\title{
Compact grating interferometer for producing photoresist gratings with incoherent light
}

\author{
Daniel Post, Krzysztof Patorski, and Peitai Ning
}

\begin{abstract}
An achromatic interferometer was developed to produce 1200 -lines $/ \mathrm{mm}$ crossed-line photoresist gratings with a mercury arc light source. It is a compact reflection system of outstanding stability. Alignment procedures are described. The most stringent requirement, coplanar alignment of two folding gratings, was accomplished with the aid of a Twyman-Green interferometer. The grating interferometer produced crossed-line photoresist gratings with first-order diffraction efficiency exceeding $20 \%$.
\end{abstract}

\section{Introduction}

A special optical configuration of an achromatic grating interferometer was developed for a specific purpose: to produce crossed-line diffraction gratings in photoresist. The objective was to produce economical gratings of 1200 lines/mm using incoherent light.

The motivation for this work was improved efficiency of moire interferometry, ${ }^{1,2}$ which is an optical technique of deformation measurements of engineering bodies. This technique uses expendable molds to replicate 1200 -lines $/ \mathrm{mm}$ crossed-line sinusoidal gratings on the surface of the body. The molds previously used for the replication had shallow grooves. With photoresist the groove depth can be optimized, resulting in replicated gratings with an order of magnitude improvement of diffraction efficiency. This paper describes the means to produce such grating molds with the incoherent light of the mercury arc lamp, using its spectral output near $\lambda 406 \mathrm{~nm}$ for exposure of the photoresist.

Important benefits are inherent in this interferometer: low optical noise, compact geometry, excellent stability, easy operation, and great economy of capital equipment. Disadvantages lie in its inability to produce gratings of different frequencies and in its difficulty of initial alignment.

The development rests on the foundation of allied work. Hutley ${ }^{3}$ produced an outstanding treatment of

The authors are with Virginia Polytechnic Institute \& State University, Engineering Science \& Mechanics Department, Blacksburg, Virginia 24061.

Received 29 October 1986

0003-6935/87/061100-06\$02.00/0.

(C) 1987 Optical Society of America. interference grating production with coherent light. Weinberg and Wood ${ }^{4}$ described the related grating interferometer illustrated in Fig. 1; Leith and his colleagues analyzed its performance with incoherent light in elegant detail. ${ }^{5-7}$ They show for the special case of symmetry where $L_{1}=L_{2}$ and gratings $A, B$, and $B^{\prime}$ have frequencies in the exact ratio $1: 2: 2$, respectively, that an interference grating is formed in recording plane $P$ by the intersecting beams. Ideally, when alignment errors are zero, the frequency of the interference grating at $P$ is equal to that of grating $B$, and there are no restrictions on coherence-any extended source of any spectral width can be used.

This paper describes a grating interferometer of similar optical properties, but one that utilizes reflection gratings. The reflection gratings permit an especially compact design, and they are commercially available with substantially higher diffraction efficiencies than corresponding transmission gratings. In addition to the schematic description of the interferometer and its properties, this paper discusses practical aspects of its alignment and performance.

\section{Configuration}

A grating interferometer was constructed with the optical arrangement illustrated schematically in Fig. 2. It is a folded version of the transmission system (Fig. 1 ), but with light paths skewed with respect to the $z$ axis. The entrance grating $A$ is a sinusoidal holographic grating of 600 lines $/ \mathrm{mm}$ and the folding gratings $B$ and $B^{\prime}$ are ruled blazed gratings of 1200 lines/ $\mathrm{mm}$. The size of each grating is $58 \times 58 \mathrm{~mm}$, with a smaller useful area. They are mounted on adjustable platforms in a relatively rigid boxlike structure. The platforms have screws for universal in-plane and outof-plane adjustments in a small range, plus locking means. 
The separation between the planes of the entrance and folding gratings is $\sim 300 \mathrm{~mm}$. The distance between the centers of gratings $B$ and $B^{\prime}$ is $\sim 150 \mathrm{~mm}$. The angular separation between the +1 and -1 orders is $\sim 28^{\circ}$ and the incidence angle $\phi$ is $\sim 10^{\circ}$ below the $z$ axis.

An interference grating of 1200 lines $/ \mathrm{mm}$ is formed in the recording plane $P$. The photoresist gratings are made on $75-\times 75-\mathrm{mm}$ glass plates coated with positive photoresist. For accurate positioning, the plate is installed in a steel frame which in turn is engaged on the interferometer frame against three balls and three pins. To produce crossed-line gratings, a first exposure is made, the steel frame carrying the photoresist plate is rotated $90^{\circ}$ and a second exposure is made on the same plate.

The light source is a $250-\mathrm{W}$ high pressure mercury arc lamp, filtered for $\lambda 406 \mathrm{~nm}$. An interference filter with a half-intensity width of $\sim 10 \mathrm{~nm}$ and an efficiency of $\sim 40 \%$ is used. A collimating lens of $350-\mathrm{mm}$ focal length is used. With its finite dimensions, the source subtends an angle of $\sim 9 \mathrm{mrad}$ in planes parallel to the grating lines and $7 \mathrm{mrad}$ in perpendicular planes.

\section{Theoretical Properties}

Like its transmission counterparts, an ideal instrument (with perfect gratings in the exact ratio of $1: 2$ and no alignment errors) produces a full contrast interference grating in plane $P$ with an intensity distribution of pure two-beam interference. The result is independent, too, of the skew angle of illumination $\phi$. The depth of fringe localization near plane $P$ depends primarily on the spatial coherence and decreases markedly as the source size increases. Optical noise, i.e., random spatial modulation of the intensity distribution, is characteristically low for this and all incoherent interferometers, relative to coherent systems.

\section{Alignment Requirements, Properties}

With incoherent light, two basic conditions must be fulfilled to realize full-contrast fringes in plane $P$. First, for any ray incident on grating $A$, the two divided rays traveling paths $A B P$ and $A B^{\prime} P$ must intersect at the recording plane $P$. Second, the path lengths of these divided rays must be equal; the diffraction angles and path lengths change with wavelength, but for any wavelength the two path lengths must be equal.

The second condition translates into this stringent requirement: gratings $B$ and $B^{\prime}$ must be coplanar. With gratings $B$ and $B^{\prime}$ coplanar, the first condition is automatically satisfied when the lines of the three gratings are mutually parallel. This is not a stringent requirement, however, since compensation is possible. Let the lines of gratings $A$ and $B$ remain parallel, while grating $B^{\prime}$ is rotated in its plane through a small clockwise angle (as viewed from the right). Then, components of a common ray will not intersect in plane $P$, but instead the ray from $B^{\prime}$ will fall below the ray from $B$. If grating $A$ is then rotated clockwise, the ray from $B$ will move down in plane $P$ while the ray from $B^{\prime}$ will move up. They will meet when the rotation of $A$ is half

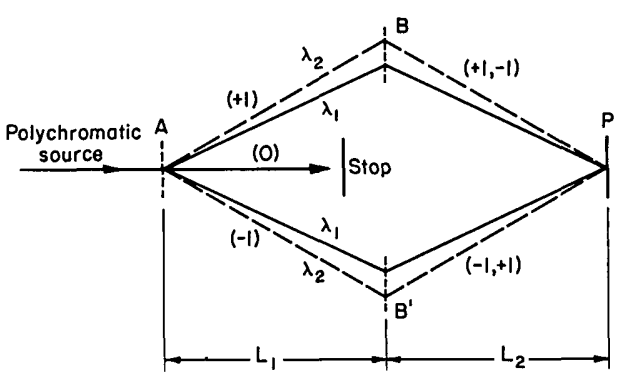

Fig. 1. Optical arrangement of a symmetrical grating interferometer with transmission gratings. Numbers in parenthesis are diffraction orders of rays. An interference grating is localized in recording plane $P$.

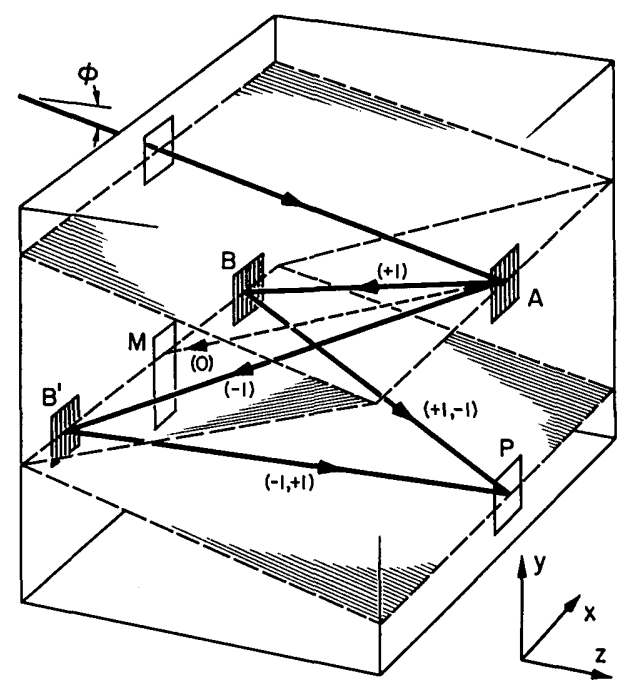

Fig. 2. Optical configuration of grating interferometer with reflection gratings. Grating frequencies are $F_{A}=600$ lines $/ \mathrm{mm}, F_{B}=F_{B^{\prime}}$ $=1200$ lines $/ \mathrm{mm}$. An interference grating of $1200 \mathrm{lines} / \mathrm{mm}$ is formed in recording plane $P$.

that of $B^{\prime}$. This is true to a first-order approximation, but since the grating misalignments contemplated here are extremely small, the approximation is adequate. Accordingly, in-plane misalignment of gratings $B$ and $B^{\prime}$ can be compensated by an adjustment of grating $A$.

\section{A. Location of Plane $\boldsymbol{P}$}

The next question is the location of the recording plane. This is the plane of fringe localization, the plane containing the crossing points of each pair of rays from $B$ and $B^{\prime}$. Full-contrast fringes are formed in this plane, but the fringes rapidly lose contrast away from the plane. Again, two factors are dominant.

One is the grating frequencies. The gratings used in the interferometer are not in the exact frequency ratio 1:2. Instead, the ratio is 1:1.9991, as determined by measurements. Consequently, the distance from the plane of $B$ and $B^{\prime}$ to plane $P$ is increased by $0.25 \mathrm{~mm}$, compared with the case of a 1:2 ratio. Plane $P$ must lie $0.25 \mathrm{~mm}$ behind the plane of grating $A$. 


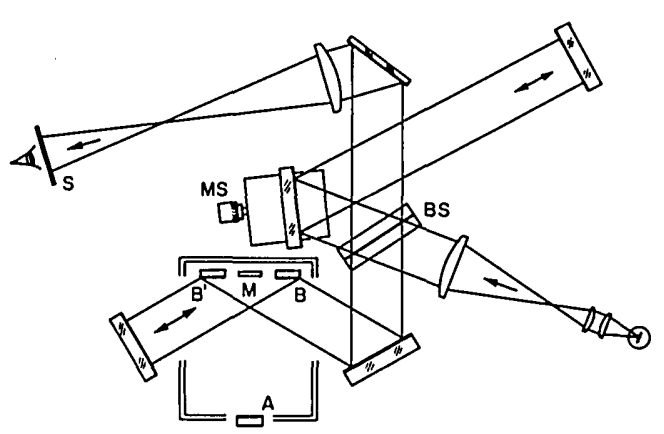

Fig. 3. Twyman-Green interferometer for guiding the adjustment of gratings $B$ and $B^{\prime}$ into a common plane: $B S$, beam splitter and compensator plate; $M S$, micrometer stage; $S$, screen.

This also influences the frequency $F_{p}$ of the interference grating in plane $P$. Assuming perfect alignment of gratings $A, B$, and $B^{\prime}$, and assuming the absolute values of grating frequencies are $F_{B}=F_{B^{\prime}}=1200$ lines/ $\mathrm{mm}$ and $F_{A}=1200 / 1.9991$, calculation yields $F_{p}=$ 1199.5 lines/mm.

The other dominant factor is out-of-plane rotation. Any tilt of grating $A$ relative to the plane of gratings $B$ and $B^{\prime}$ causes changes in locations of the crossing points of corresponding rays coming from $B$ and $B^{\prime}$. As a result, the plane of localization tilts and translates (in the $z$ direction) when $A$ is tilted.

To obtain full fringe contrast in practice, it is not necessary to provide fine adjustments on both grating $A$ and plane $P$. Either is sufficient. Coarse adjustments were provided for plane $P$ and fine adjustments were provided only on $A$. Then, by adjusting $A$, the plane of optimum fringe localization could be moved into coincidence with $P$.

\section{Alignment Procedure}

The alignment was done after final assembly of the interferometer. The structure of the interferometer hampered access of auxiliary optical elements used for alignment and special systems were required. The adjustments were made in the following sequence:

(1) Grating $B$ was locked in position.

(2) Grating $B^{\prime}$ was adjusted coplanar with $B$.

(3) Plane of $P$ was adjusted parallel to $B$ and $B^{\prime}$.

(4) Lines of $B^{\prime}$ were adjusted parallel to lines of $B$; in-plane rotation of $B^{\prime}$ was locked.

(5) Coplanar adjustment of $B$ and $B^{\prime}$ was improved and $B^{\prime}$ was locked.

(6) Grating $A$ was adjusted to produce a full-contrast interference grating in plane $P$; out-of-plane adjustments of $A$ were locked.

\section{A. Coplanar Adjustment (Steps 2 and 5)}

The tolerance on the equality of optical path lengths is very small when a temporally incoherent source is used. The coplanar adjustment of $B$ and $B^{\prime}$ must be made within a few wavelengths if a high contrast interference grating is to be produced in the recording plane $P$. The adjustment is distinctly different from that of the transmission interferometer of Fig. 1, for which the equality of path lengths is more easily achieved.

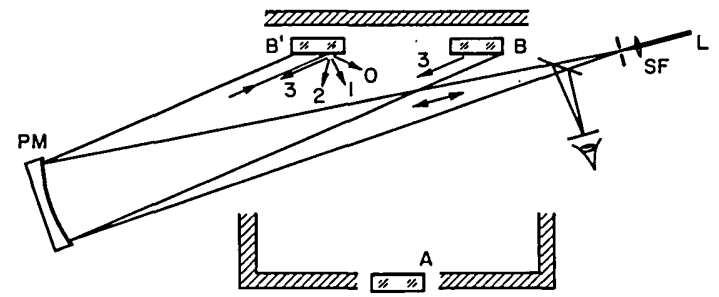

Fig. 4. Autocollimation to adjust parallelism of lines of gratings $B$ and $B^{\prime}$. The third order diffraction beams were returned to the source: $P M$, parabolic mirror of 1.2 -m focal length; $L$, laser beam, $\lambda 514 \mathrm{~nm} ; S F$, spatial filter.

Figure 3 shows the method used for assessing and guiding the alignment of $B^{\prime}$. A Twyman-Green interferometer was used to sense the imbalance of path lengths introduced by out-of-plane positions of $B^{\prime}$. The unusual configuration was prompted by limitations of access. Calculation shows that the sensitivity to out-of-plane positions of $B^{\prime}$ is $\lambda / 2$ per fringe order when the angle of incidence on the gratings is $60^{\circ}$.

Different light sources were used in the course of the adjustment, sequentially: a laser, a low pressure mercury lamp, a high pressure mercury lamp, and an incandescent lamp. This allowed progressively closer approaches to finding the zero-order white-light fringes in gratings $B$ and $B^{\prime}$; the length of the reference beam was altered (by adjusting the micrometer stage $M S$ ) until it matched the active path length. The adjustment of $B^{\prime}$ was complete when the closest approach to a zero-order fringe appeared simultaneously over gratings $B$ and $B^{\prime}$. In fact, it was found that the grating surfaces were not optically flat, the greatest deviation from flatness occurring near one corner of grating $B$. The final adjustment brought the centers of both gratings into a common plane and the corners within 1.5 wavelengths (white light) of the common plane.

We must note parenthetically that we cannot be certain the instrument has maintained this degree of precision, but no degradation of performance has been observed in three months.

Figures 2 and 3 show a plane mirror $M$ located between gratings $B$ and $B^{\prime}$. This mirror was used in the alignment procedure only (step 3) and it was blocked by a black obstacle thereafter. Mirror $M$ was adjusted parallel to $B$ with the Twyman-Green interferometer.

\section{B. Parallelism of $\boldsymbol{P}$ (Step 3)}

A semitransparent mirror was installed in the frame used to carry the photoresist plate. Adjustments were made to align this semitransparent mirror parallel to mirror $M$ by autocollimation.

\section{Parallelism of Grating Lines (Step 4)}

Parallelism of the lines of gratings $B$ and $B^{\prime}$ was adjusted by the autocollimation method of Fig. 4 . Light from the third diffraction order was returned to the source from gratings $B$ and $B^{\prime}$. It was redirected by a beam splitter and viewed on a screen. Two dots of light appeared on the screen, and they were brought 


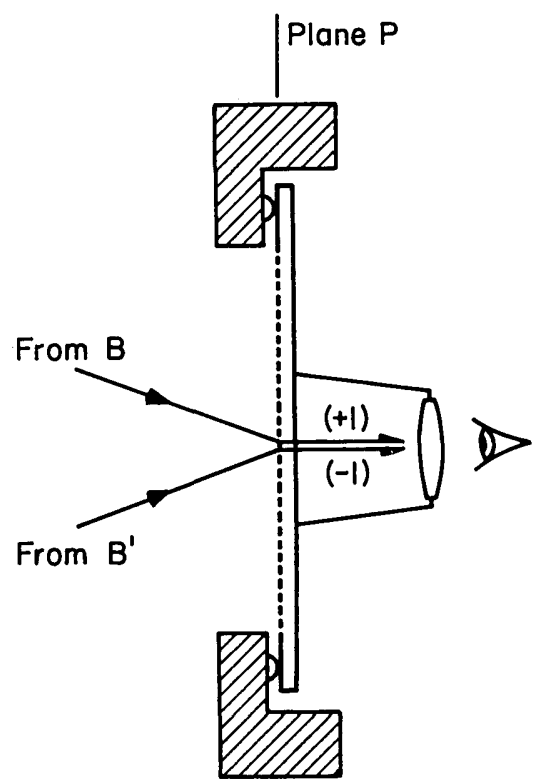

Fig. 5. Interference of the beams diffracted by a 600 -lines $/ \mathrm{mm}$ auxiliary grating senses the localization and contrast of the interference grating.

together by in-plane rotation of grating $B^{\prime}$. Then the rotation was locked. The combined conditions of superimposition of the dots and parallelism of $B$ and $B^{\prime}$ assure parallelism of the grating lines. Note that accurate collimation, i.e., accurate location of the spatial filter, is required for this method.

\section{Adjustment of Grating A (Step 6)}

Grating $A$ must be adjusted such that corresponding rays along the two paths cross in the fixed plane $P$. This results in full-contrast interference fringes in plane $P$. The adjustment was accomplished as follows.

An auxiliary transmission grating of 600 lines $/ \mathrm{mm}$ was installed in the fixture defining plane $P$. As illustrated in Fig. 5, light from $B$ and $B^{\prime}$ was diffracted by the auxiliary grating in its +1 and -1 diffraction orders, respectively. These diffracted beams combined to produce an optical interference pattern (or moire pattern) which was readily viewed with a magnifier focused on plane $P$. The fringes had good contrast where corresponding rays from $B$ and $B^{\prime}$ met on the surface of the auxiliary grating, and they had poorer contrast where the rays met ahead of or behind the surface. Out-of-plane adjustments of grating $A$ were made to progressively improve the contrast. For each of these adjustments, an in-plane rotation adjustment of grating $A$ was made, too, to optimize fringe contrast. When full contrast was achieved, the plane of the auxiliary grating coincided with the plane of localization of the interference grating.

The fringe contrast was subjective and final adjustments were made with an objective method. When the interference grating coincides with the auxiliary grating, the fringes are independent of the direction of light incident on the interferometer. Where they do

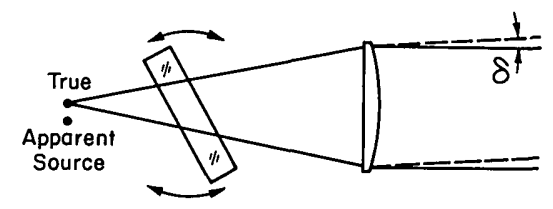

Fig. 6. Direction of illumination is oscillated through $\pm \delta$ by oscillating a thick plate.

not coincide, the fringes move as the direction of incidence is changed. To utilize this property, a thick plate was inserted in the beam diverging from the source as illustrated in Fig. 6. Its inclination was oscillated to cause an oscillation of the direction of the incoming beam. As a result, fringes viewed on the auxiliary grating oscillated about a central position, except where the auxiliary grating coincided with the plane of localization and the fringes were stationary. Further adjustments of grating $A$ were made to eliminate fringe motion. Their motion could not be eliminated throughout the entire extent of the interference grating, however. Instead, the final adjustment achieved zero fringe movement at the center of the field and minimum movement at the corners. Warpage of the surface of localization, or the surface of the auxiliary grating, would account for the imperfect superimposition. Nevertheless, fringes appeared in excellent visual contrast. This established the recording plane for exposing photoresist plates.

The recording plane coincided sufficiently well with the localization of the interference grating. The depth of localization was small, but it was enough to accommodate the surface warpage of common $3-\mathrm{mm}$ thick glass plates. The depth of localization can be increased by reducing the effective size of the source.

\section{Results}

Crossed-line phase gratings were exposed in the recording plane on Shipley Microposit S-1400 photoresist. The photoresist coated plates were exposed in the interferometer, rotated $90^{\circ}$, and exposed again. After optimization for diffraction efficiency, the exposures were $3.7 \mathrm{~min}$ each for the two orthogonal exposures. The maximum local exposure, $J_{\max }$, occurred at crossing points where each of the interference gratings had its maximum intensity. An additional uniform preexposure $J_{0}$ was used, where $J_{0}=0.7 J_{\max }$. Immersion development for 2 min was used with Shipley Microposit 352 developer.

Optimization of the first-order diffraction efficiency was done in the second-order Littrow configuration (oblique incidence such that the emergent first order is normal to the grating), which corresponds to the configuration of moire interferometry. Absolute diffraction efficiency was measured as the first-order emergent light intensity (normal to the grating) divided by the incident light intensity. It was measured at 514$\mathrm{nm}$ wavelength, with polarizations parallel and perpendicular to the plane of incidence ( $\perp$ and $\|$ to the lines of the grating, respectively). 
For the stated exposures, and with a reflective coating of evaporated aluminum applied to the grating, the diffraction efficiencies were $E_{1 \|}=22 \% ; E_{2 \|}=19 \% ; E_{1 \perp}$ $=10 \%$; and $E_{2 \perp}=9 \%$. Here, subscripts 1 and 2 refer to the gratings of the first and second exposures, respectively, and $\|$ and $\perp$ refer to polarizations with respect to the plane of incidence. To achieve equal diffraction efficiencies of the orthogonal gratings, the second exposure time could be increased or the preexposure could be increased.

For normal incidence, the efficiencies are within $1 \%$ of the second-order Littrow efficiencies. The diagonal first orders exhibit 5\% efficiency. It is interesting to note that $\sim 80 \%$ of the incident light emerges in the first diffraction orders of the crossed-line grating.

Details of the grating surface are visible in a scanning electron micrograph, Fig. 7. The crossed-line phase grating is comprised of orthogonal arrays of hills and holelike valleys. The ridgeline through a row of hills has an undulating height that is essentially sinusoidal. Similarly, a section through a row of valleys has a lower height, but a corresponding sinusoidal undulation.

The uniformity of lines of the gratings produced by the achromatic interferometer was assessed by comparing it to a virtual reference grating of 2400 lines $/ \mathrm{mm}$ in a coherent light moire interferometry system. ${ }^{1,2}$ The system reveals in-plane uniformity of the test grating, independent of out-of-plane features. The virtual reference grating was adjusted for a null field using a 1200 -lines $/ \mathrm{mm}$ commercial ruled grating of high quality. When the test grating was installed in its place, the moire interference fringes were straight and parallel in the central $25-\mathrm{mm}$ square zone. Their frequency was -0.8 fringes $/ \mathrm{mm}$, indicating that the frequency of the test grating was 0.4 lines $/ \mathrm{mm}$ less than that of the ruled grating. When the virtual reference grating was adjusted for the best null field, a variation of only one fringe appeared in a region $\sim 40 \times 45 \mathrm{~mm}$. Overall, the maximum error was two fringes, which appeared in one corner of the test grating. This result corresponds to an observation mentioned in Sec. V. A, where a surface aberration was detected in grating $B$ in the same corner. Note that this test has double sensitivity, relative to a moire test with a 1200 -lines $/ \mathrm{mm}$ reference grating. For the intended purpose of deformation measurements of engineering bodies by moire interferometry, these gratings are fully satisfactory.

The achromatic grating interferometer is remarkably stable. While it is supported on a vibration isolation table, no jitter of the interference grating can be detected when the isolation is deactivated. No readjustment is required over long periods of timeweeks - and then the in-plane rotation of grating $A$ is tuned slightly for optimum fringe contrast.

\section{Discussion}

The diffraction efficiency of the shallow crossed-line specimen gratings previously used in moire interferometry $^{1,2}$ was $\sim 2 \%$. With the optimized depth of photoresist gratings, an order of magnitude increase is

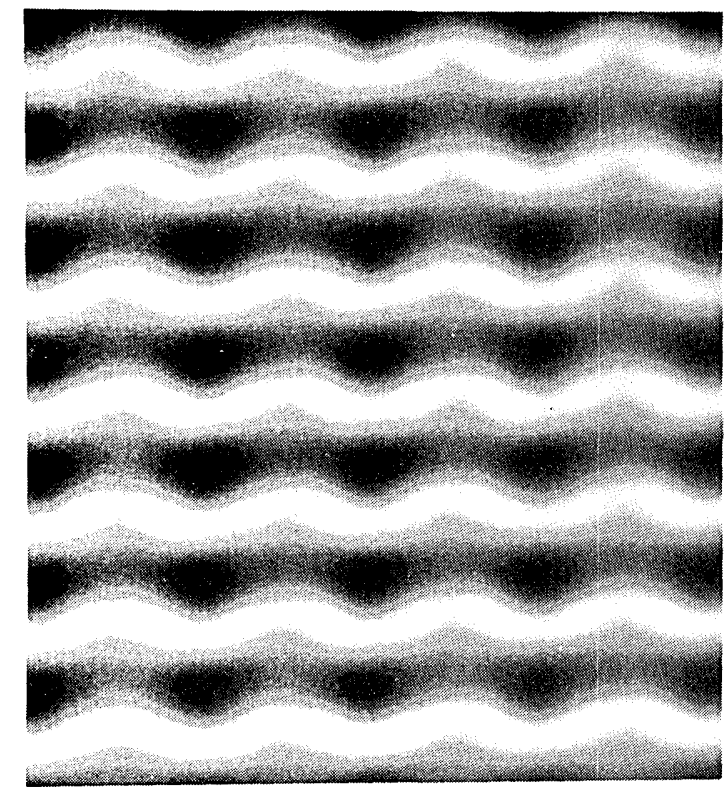

Fig. 7. An SEM image of the crossed-line photoresist grating. The grating is viewed from above at an angle of $\sim 45^{\circ}$.

available. The direct consequence is that moire interferometry apparatus can use laser light sources of proportionally lower power.

Contrary to theory, the results indicate that the surface of fringe localization is not precisely plane, but slightly warped. The greatest departure from a plane occurred in the region corresponding to the largest error of flatness of grating $B$. The warpage of the surface of localization is attributed to aberrations of the gratings, especially errors of flatness, and to residual errors of alignment.

Experiments were performed without the interference filter in the system. Then, the photoresist responds to the 366-, 406-, and 436-nm wavelengths of the mercury spectrum. There is a corresponding decrease of exposure time by 1 order of magnitude. The results showed equally high diffraction efficiency in the central region, but zones of lower efficiency near the edges of the grating. The results seem to be more sensitive to the aberrations of the interferometer, and it is suggested that a system with higher quality gratings could be used successfully without the filter. The effective width of the interference grating is decreased because of the finite width of gratings $B$ and $B^{\prime}$; these gratings are positioned to intercept the beams diffracted from the entrance grating in 406-nm wavelength, but for other wavelengths, the beams are not fully intercepted by the gratings.

With the optical configuration of Fig. 2. an equivalent to the photoresist preexposure can be achieved automatically. Normally mirror $M$ is covered, but if it is uncovered, light from the zero order of the entrance grating strikes the mirror and reflects into the recording plane of the photoresist plate. This light can provide the preexposure energy.

An interesting opportunity exists for producing photoresist gratings that are substantially larger than 


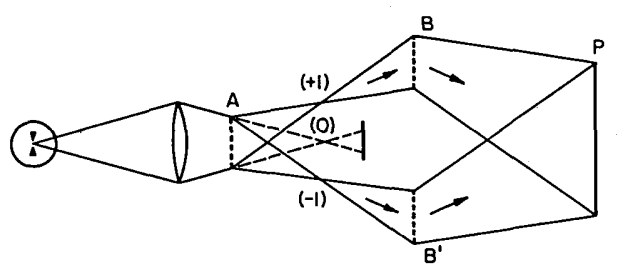

Fig. 8. Converging input beam can produce a larger interference grating in recording plane $P$. Transmission or reflection systems can be used.

gratings $A, B$, and $B^{\prime}$. The scheme is illustrated in Fig. 8 for a transmission configuration, but it applies to reflection too. With convergent illumination on grating $A$ as shown, the interference grating in plane $P$ is 3 times larger than $A$ and $B$, with 9 times greater area. Its frequency is equal to that of grating $B$. For an ideal system-a system free of grating errors and alignment errors-the interference grating formed at $P$ is independent of the direction of incoming light, which means that a convergent or divergent input beam is applicable. The configuration seems practical when gratings of excellent surface figure are used in the instrument. In a related experiment, the lens in Fig. 8 was not present and light beams diverged throughout the interferometer to produce an interference grating at $P$ that was 1.5 times larger than grating $B$. The reflective system of Fig. 2 was used. The resulting contrast of the interference grating was excellent, but the deviation from straight, uniformly spaced lines was greater than that obtained with collimated light. Again, the reason appears to be errors of gratings in the achromatic interferometer.

\section{Conclusions}

Crossed-line phase gratings of 1200 lines $/ \mathrm{mm}$ can be made efficiently in photoresist with an achromatic grating interferometer. A reflection system using a mercury arc light source is effective. Stringent alignment requirements were implemented with a Twyman-Green interferometer and autocollimation. The interferometer is extremely stable. Capital costs are small relative to comparable coherent light systems.
Photoresist crossed-line gratings exhibited over $20 \%$ diffraction efficiency in reflection. Uniformity of the grating lines depends on the aberrations of gratings in the instrument, notably their surface flatness. The depth of the zone of fringe localization in the recording plane depends on the size of the source; for this instrument, the depth was sufficient to accommodate the surface warpage inherent in common glass substrates. An optical configuration is suggested to produce interferometric gratings in the recording plane that are larger than the gratings in the instrument.

M. C. Hutley and his associates at the National Physical Laboratory, Teddington, England, kindly taught one of us (D.P.) important aspects of producing photoresist gratings in coherent light. E. N. Leith kindly provided valuable assistance in personal correspondence. This research was sponsored by the National Science Foundation under grant MEA-8317879 and the Office of Naval Research under grant N0001486-K-0255. This outstanding support is warmly appreciated and gratefully acknowledged.

Krzysztof Patorski is a visiting scientist from Warsaw Technical University, Poland. Peitai Ning is a visiting scholar from the Harbin Shipbuilding Engineering Institute, China.

\section{References}

1. D. Post, "Moire Interferometry at VPI and SU," Exp. Mech. 23, 203 (1983).

2. D. Post, "Moire Interferometry," in Handbook of Experimental Mechanics, A. S. Kobayashi, Ed. (Prentice-Hall, Englewood Cliffs, NJ, 1986).

3. M. C. Hutley, Diffraction Gratings (Academic, New York, 1982).

4. F. J. Weinberg and N. B. Wood, "Interferometer Based on Four Diffraction Gratings,” J. Sci. Instrum. 36, 227 (1959).

5. E. N. Leith, G. Swanson, and S. Leon, "Construction of Diffractive Optical Elements in Non-Coherent Light," Proc. Soc. PhotoOpt. Instrum. Eng. 503, 3 (1984).

6. G. J. Swanson, "Broad-Source Fringes in Grating and Conventional Interferometers," J. Opt. Soc. Am. A 1, 1147 (1984).

7. B. J. Chang, R. C. Alferness and E. N. Leith, "Space-Invariant Achromatic Grating Interferometers: Theory," Appl. Opt. 14, 1592 (1975). 